\title{
In-Fibre Diffraction Grating Based Beam Steering for Full Duplex Optical Wireless Communication
}

\author{
Guoqing Wang ${ }^{1}$, Usman Habib ${ }^{1}$, Zhijun Yan ${ }^{2,3}$, Nathan J. Gomes ${ }^{1}$, Lin Zhang ${ }^{3}$, and Chao Wang ${ }^{1 *}$ \\ ${ }^{1}$ School of Engineering and Digital Arts, University of Kent, Canterbury, United Kingdom CT2 7NT \\ ${ }^{2}$ School of Optical and Electronic Information (SOEI), Next Generation Internet Access National Engineering Laboratory \\ (NGIAS), Huazhong University of Science and Technology, Wuhan, 430074, China \\ ${ }^{3}$ Aston Institute of Photonic Technologies, Aston University, Birmingham, United Kingdom B4 7ET \\ *e-mail: c.wang@kent.ac.uk
}

\begin{abstract}
A novel approach to achieve wavelength controlled optical beam steering using a $45^{\circ}$ tilted fiber grating (TFG) for full-duplex indoor optical wireless transmission is proposed and experimentally demonstrated for the first time. The $45^{\circ}$ TFG functions as an in-fiber passive diffraction device for wavelength steered light emission and reception, which enables full-duplex optical wireless transmission. The unique advantages of using an in-fiber TFG device for beam steering include high diffraction efficiency, low cost, compactness and inherent compatibility with existing fiber links. In a proof-of-concept experiment, $1.4 \mathrm{~m}$ freespace full-duplex transmission has been demonstrated with data rate of $9.6 \mathrm{~Gb} / \mathrm{s}$ per beam using $2.4 \mathrm{GHz}$ bandwidth signals.
\end{abstract}

Keywords-Beam steering, diffraction gratings, optical wireless communication, tilted fiber gratings

\section{INTRODUCTION}

Nowadays drastically increasing numbers of wireless communication devices, such as smartphones, smartwatches, laptops, tablets and other wireless sensors connect in home area networks. There is a real demand for higher data transmission speed, a need to overcome radio spectrum congestion and to give the users better experience for easy internet access with unobstructed service. However, with the approaching $5 \mathrm{G}$ wireless networks, the available radio frequency (RF) bandwidth has nearly hit limit due to the increasing demanding of high speed wireless transmission applications [1].

A substitute for conventional radio frequency wireless transmission is free-space optical wireless communication [2], which takes advantages of the huge bandwidth of optical carriers and mature transmission technology developed in long-haul optical fiber communications. Free-space optical wireless communication provides extremely wide bandwidth compared to RF spectrum, tens of thousands of times wider than conventional radio wireless communication [3]. In addition, free-space optical wireless communication lies in the unlicensed spectrum within a wide range of wavelength bands, thus permitting adequate room for spectrum sharing with low-cost. Other advantages of optical wireless communication include physically ensured security, anti-electromagnetic interference, low power consumption, spatial diversity and low cost deployment.

Visible light communication (VLC) is one of the commonly used solutions for free-space optical wireless communication.

This work was supported in part by the EU H2020 RAPID Project under grant 643297 and in part by the EU FP7 Marie-Curie Career Integration Grant under grant 631883 .
But its data rate is normally limited to hundreds of Mbps due to the limitation of low speed of modulation of the light emitting diodes (LED) used [4]. An alternative solution for optical wireless communication is to use infrared spectrum, with its unique benefits of wide bandwidth, high speed, easy access and low cost due to readily available fiber communication devices, such as infrared laser, optical fiber, photodetectors. In addition, infrared wireless communication offers higher link power budget due to the relaxed eye-safety regulations in this wavelength band.

In infrared wireless communication systems, a beam steering device is a key element to scan the laser beam for large area coverage. Several attempts are made to meet the need of beam steering applications. Conventionally, mechanical beam steering solutions have attracted great attention and were extensively studied due to their merits of high resolution and relatively large steering angle $[5,6]$. However, they suffer from the difficulties of relatively low steering speed and bulky size. Therefore, to facilitate practical deployment of in-door infrared wireless communication systems, a fast beam-steering device with compact size is of high demand. To tackle these challenges, various proposals for mechanical-free beam steering have been presented, such as the use of diffraction gratings [7], polymer polarization grating [8], photonic crystal laser [9], and optical phased array [10]. Most recently, we have demonstrated an allfiber wavelength-tuning-based passive optical beam steering approach [11]. A $45^{\circ}$ tilted fiber grating (TFG) was employed as a highly efficient, low cost and compact in-fiber diffractive device for wavelength controlled beam steering in indoor optical wireless transmission. The TFG-based solution offers inherent compatibility with existing fiber links and eliminates the significant coupling loss between fibers and free-space diffraction gratings. However, only downlink wireless transmission from the access point to the remote users was demonstrated.

In this work, we investigate and demonstrate, for the first time, a full-duplex bidirectional in-door infrared wireless transmission system using a $45^{\circ} \mathrm{TFG}$ for laser beam steering. Beam steering for full-duplex transmission is made possible due to the fact that the tilted grating structure in the TFG allows it to function as a light receiver as well. It has the advantage of low cost, high diffraction efficiency, and stable and simple beam steering in full-duplex bidirectional indoor optical wireless communication system. TFG's capability of light collection in 
uplink transmission has been verified. We also carried out a proof-of-concept experiment where full-duplex bidirectional optical wireless transmission within $1.4 \mathrm{~m}$ has been demonstrated with an aggregate data rate of $9.6 \mathrm{~Gb} / \mathrm{s}$ per infrared beam using orthogonal frequency-division multiplexing (OFDM) intensity modulation.

\section{PRICIPLE OF $45^{\circ}$ TILTED FIBER GRATING}

Compared to a normal fiber Bragg grating, a TFG's grating structure has a tilted angle with respect to the fiber axis, which endows it with unique optical properties. For example, commonly used TFGs have a small tilted angle (less than $9^{\circ}$ ), where the transmitted light in the fiber core region will be coupled into both backward-propagating core mode and contrapropagating cladding modes, resulting in multiple resonances at the transmission spectrum. This feature makes small-angle TFGs a good candidate for sensing application [12]. On the other hand, for a $45^{\circ} \mathrm{TFG}$, the forward propagating light is coupled from core mode into radiation modes owing to its largely tilted facet angle, leading to direct lateral diffraction into free space. Following its original application as an in-line fiber polarizer [13], the $45^{\circ} \mathrm{TFG}$ has found rich application in various fields [14], such as optical spectrum analysis [15], spectrally-encoded optical imaging $[16,17]$, and optical beam steering [11].

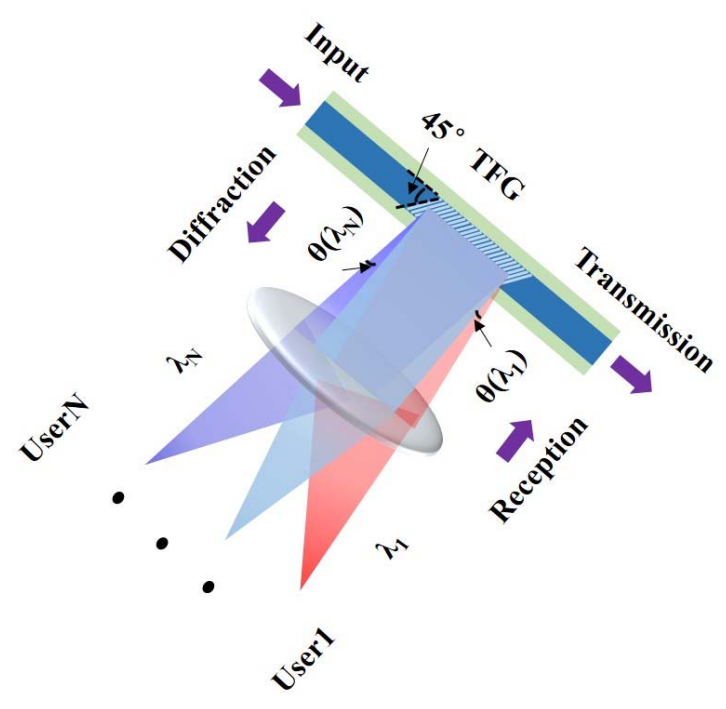

Fig. 1: The structure of a $45^{\circ} \mathrm{TFG}$ and its application for passive beam steering.

In our proposed full-duplex optical wireless transmission system, the $45^{\circ} \mathrm{TFG}$ serves as an in-fiber diffraction grating for wavelength-controlled laser beam steering. At the same time, the $45^{\circ} \mathrm{TFG}$ also collects uplink light signal from remote users. The structure of a $45^{\circ}$ TFG is shown in Fig. 1. When the broadband incident light propagates through the $45^{\circ} \mathrm{TFG}$, light with different wavelengths is laterally diffracted into free space with different angles. The tiled angle of $45^{\circ}$ is particularly selected as it ensures a maximum angular dispersion for a given wavelength [15], thus guaranteeing a stronger wavelength-to-angle mapping for beam steering. The angular dispersion of $45^{\circ}$ a TFG is given by [15]

$$
D=\frac{d \theta(\lambda)}{d \lambda}=\frac{1}{\lambda}
$$

where $\lambda$ is the wavelength of incident light and $\theta$ is the angle of wavelength-dependent lateral diffraction. In our case, when $\lambda$ is selected as $1550 \mathrm{~nm}$, the theoretical angular dispersion value for a $45^{\circ} \mathrm{TFG}$ is $0.037^{\circ} / \mathrm{nm}$ according to Eq. (1). Considering the refraction at the interface between fiber cladding and air when the light is emitted into air, the off-fiber angular dispersion is $0.054^{\circ} / \mathrm{nm}$. This makes the $45^{\circ}$ TFG a good candidate for wavelength-controlled infrared laser beam steering.

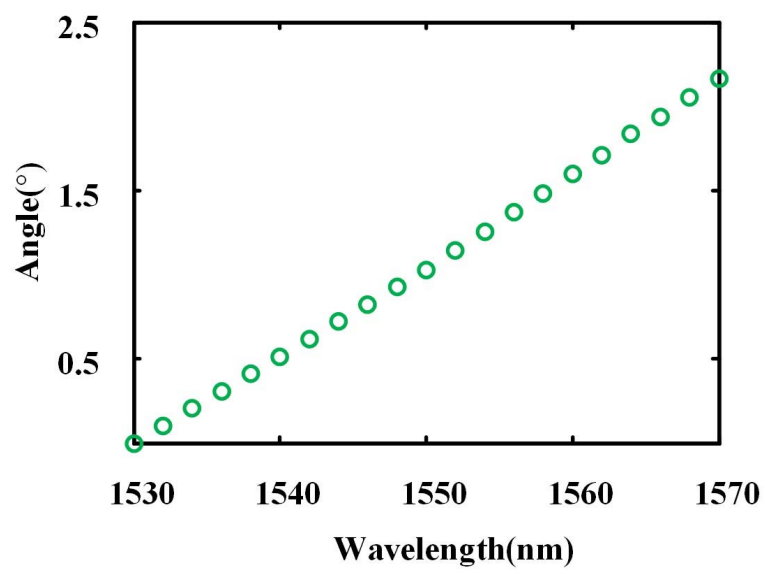

Fig. 2: Measured off-fiber angular dispersion of the $45^{\circ}$ TFG across wavelength range between 1530 and $1570 \mathrm{~nm}$.

\section{EXPERIMENT AND RESULTS}

A $24 \mathrm{~mm}$ long $45^{\circ} \mathrm{TFG}$ was fabricated for this experiment using the standard UV-light imprinting and phase mask technique. The phase mask is tilted at an angle of $33.7^{\circ}$ with respect to the fiber axis to form the required $45^{\circ}$ slanted grating fringes. Basic performance of the fabricated $45^{\circ} \mathrm{TFG}$ was tested first. The measured off-fiber beam divergence angle with respect to fiber axis across a wide wavelength range from 1530 to $1570 \mathrm{~nm}$ is shown in Fig. 2. The angular dispersion is estimated to be $0.053^{\circ} \mathrm{nm}$, which shows a good agreement between the experimental and theoretical values.

Light scattering from the $45^{\circ} \mathrm{TFG}$ is strongly polarization dependent. Only an s-polarized light beam can be emitted from the fiber core. By properly controlling the polarization of incident light, a high diffraction efficiency $(>93 \%)$ is achieved. In addition, free-space link loss between input of TFG and out of receiving fiber collimator has been measured. At a selected wavelength of $1540 \mathrm{~nm}$, the measured free-space loss for downlink transmission is $7.3 \mathrm{~dB}$, which is $6 \mathrm{~dB}$ less than that of the normal diffraction grating based beam steering system [3]. Uplink transmission is also tested by launching laser beam from the remote user and measuring the collected at the output of TFG. Uplink free-space loss is measured to be $7.8 \mathrm{~dB}$.

Fig. 3 shows the diagram of duplex bidirectional free-space indoor wireless communication system using the $45^{\circ} \mathrm{TFG}$ as infiber beam steering device. A wideband orthogonal frequencydivision multiplexing (OFDM) signal with $2.4 \mathrm{GHz}$ bandwidth 


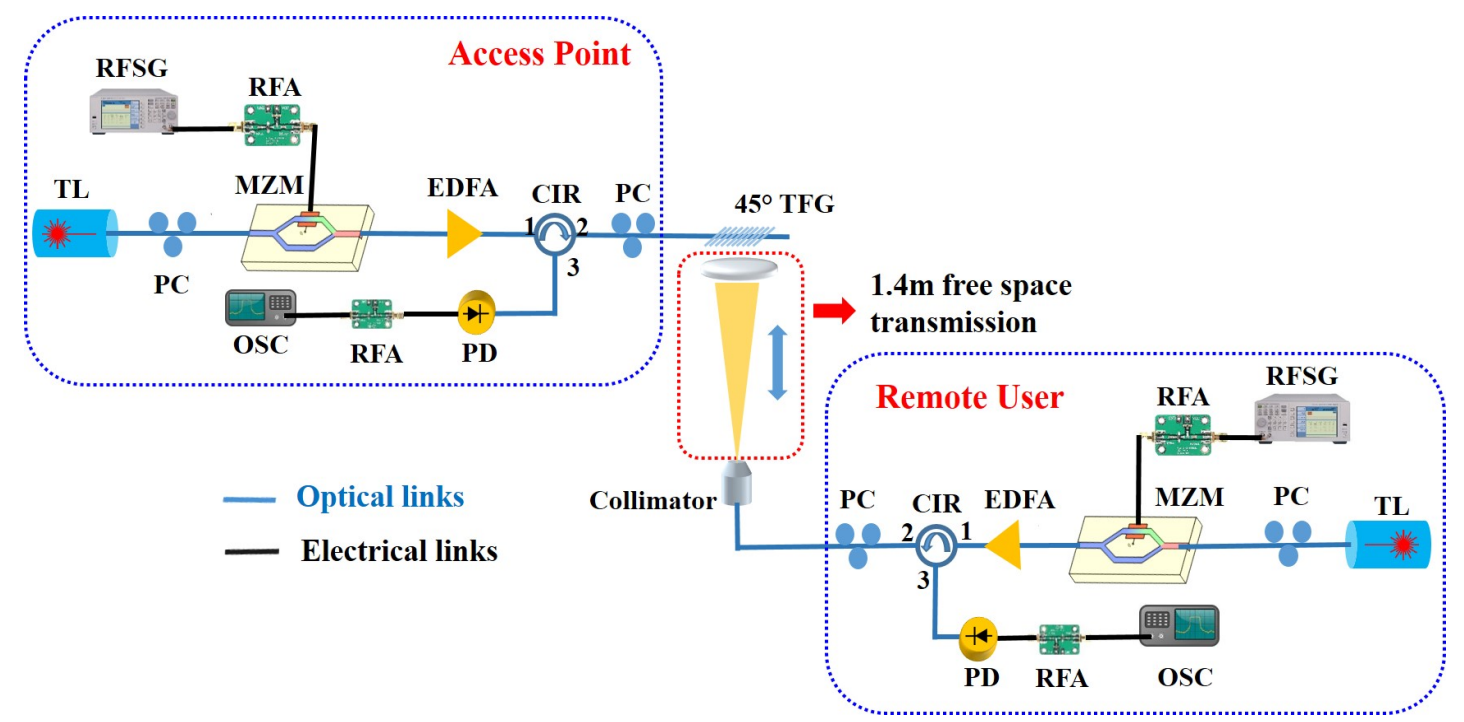

Fig. 3. Schematic of $45^{\circ}$ TFG based beam steering system for full-duplex bidirectional indoor optical wireless communication. TL: Tunable Laser; PC: Polarization Controller; MZM: Mach-Zehnder Modulator; RFSG: Radio Frequency Signal Generator; RFA: Radio Frequency Amplifier; EDFA: Erbium Doped Fiber Amplifier; CIR: Circulator; $45^{\circ} \mathrm{TFG}$ : $45^{\circ}$ Tilted Fiber Grating; OSC: Oscilloscope; PD: photodetector.

and 16-quadrature amplitude modulation (16-QAM) encoded data stream at $9.6 \mathrm{~Gb} / \mathrm{s}$ is generated on a $2 \mathrm{GHz}$ radio frequency carrier using a radio frequency signal generator (RFSG, Tektronix 7122C) with a sampling rate of $12 \mathrm{GS} / \mathrm{s}$. After amplification by a radio frequency amplifier (RFA), the 16QAM OFDM signal modulates the optical carrier using a MachZehnder modulator (MZM), which is biased at quadrature point. Scanning of optical wavelength is achieved by using a tunable laser (TL) source. An optical fiber polarization controller (PC) is used to ensure s-polarization for the incident light into the $45^{\circ}$ TFG, which guarantees the maximum diffraction efficiency. The $45^{\circ}$ TFG diffracts light of different wavelength into different directions thanks to its angular dispersion. Optical beam steering is done by tuning the wavelength of the optical carrier. In this proof-of-concept experiment, an optical wavelength of $1540 \mathrm{~nm}$ is selected to serve one remote user.
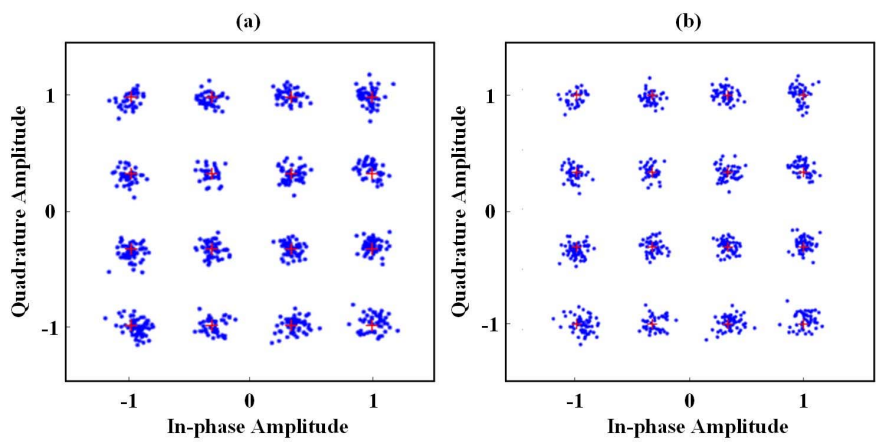

Fig. 4: Constellation of received 16-QAM OFDM signal for (a) uplink channel with 9.6\% EVM and (b) downlink channel with 9.2\% EVM. The optical wavelength is $1540 \mathrm{~nm}$ and the received optical power is $-2 \mathrm{dBm}$.

A lens set is employed to collimate and shrink the beam size. After $1.4 \mathrm{~m}$ in-door free space transmission, the laser beam is received by a remote user where a fiber collimator with numerical aperture (NA) of 0.3 is used to collect the transmitted light for the specific user. An optical circulator is used in both the access point and remote user site to allow full-duplex operation. The received light is detected by photo-detectors (PDs) with $3 \mathrm{GHz}$ analog bandwidth at the access point and the user. The recovered RF signal is amplified by a second RFA and sampled by a $100 \mathrm{GS} / \mathrm{s}$ real-time oscilloscope (OSC, Tektronix DPO72304DX). Received data is analysed using MATLAB for off-line digital data processing.

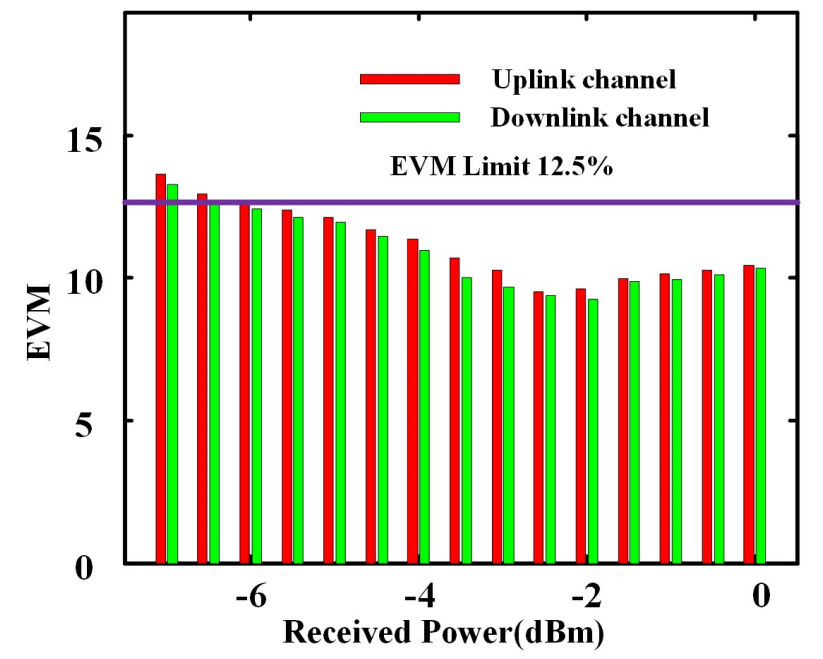

Fig. 5: The EVM performance of downlink and uplink channels with the wavelength of $1540 \mathrm{~nm}$ with received optical power from -7 to $0 \mathrm{dBm}$.

The constellations of received 16-QAM OFDM signals for uplink channel and downlink channel are shown in Figs. 4(a) and (b), respectively. Here the received optical power is $-2 \mathrm{dBm}$. The error vector magnitude (EVM) of uplink and downlink channels are estimated as $9.6 \%$ and $9.2 \%$ EVM.

Performance of our full-duplex bidirectional optical wireless transmission system is further evaluated. EVM results of both uplink and downlink channels for the received optical power 
from $-7 \mathrm{dBm}$ to $0 \mathrm{dBm}$ are shown in Fig. 5. The EVM limit for 16 -QAM is $12.5 \%$ as indicated by a purple solid line. With the increase in received optical power, the EVM of both channels are improved as expected. For higher received optical power above $-2 \mathrm{dBm}$, the EVM results for both downlink and uplink channels degrade, owing to the saturation of the RFA. Our presented system achieves indoor full-duplex bidirectional optical wireless transmission with a data rate of $9.6 \mathrm{~Gb} / \mathrm{s}$ per laser beam using a bandwidth of $2.4 \mathrm{GHz}$. The bitrate in our case is only limited by the bandwidth of our RFSG and can be easily improved by using more powerful RF signal generation hardware. Furthermore, due to the nature of strong polarization dependent diffraction, high-speed polarization modulation could be implemented to avoid the inherent DC bias drift problem in MZMs as used in this work.

\section{CONCLUSIONS}

A novel indoor full-duplex optical wireless communication system using a $45^{\circ} \mathrm{TFG}$ as in-fiber diffraction grating for passive laser beam steering is proposed and experimentally demonstrated for the first time. Bi-directional light transmission is made possible by using the $45^{\circ}$ TFG to simultaneously emit and collect light. TFG-based optical beam steering solution has the benefits of low-cost, compact, inherent fiber-compatible, polarization sensitive and high diffraction efficiency. The use of $45^{\circ}$ TFG can significantly improve the stability and efficiency of the system and reduce the physical volume by combining beam steering device and light emitter/receiver into to one single optical fiber element. 16-QAM OFDM intensity modulation has been employed to achieve data rate of $9.6 \mathrm{~Gb} / \mathrm{s}$ per beam within free space transmission of $1.4 \mathrm{~m}$. The presented system offers a promising solution for high data-rate in-door optical wireless communication with flexible beam steering capability.

\section{REFERENCES}

[1] S. Cherry, "Edholm's law of bandwidth," IEEE Spectr., vol. 41, no. 7, pp. $58-60,2004$.

[2] H. Elgala, R. Mesleh, and H. Haas, "Indoor optical wireless communication: potential and state-of-the-art," IEEE Commun. Mag., vol. 49, no. 9, pp. 56-62, 2011.

[3] C. W. Oh, Z. Cao, E. Tangdiongga, T. Koonen, "Free-space transmission with passive $2 \mathrm{D}$ beam steering for multi-gigabit-per-second per-beam indoor optical wireless networks," Opt. Express. vol.24, no.17, pp. 1921119227, 2016.

[4] Y. Wang, Y. Shao, H. Shang, X. Lu, Y. Wang, J. Yu, and N. Chi, "875$\mathrm{Mb} / \mathrm{s}$ asynchronous bi-directional 64QAM-OFDM SCM-WDM transmission over RGB-LED-based visible light communication System," in Optical Fiber Communication Conference, 2013 OSA Technical Digest Series (Optical Society of America, 2013), paper OTh1G-3.

[5] T. Matsuda, F. Abe, and H. Takahashi, "Laser printer scanning system with a parabolic mirror," Appl. Opt., vol. 17, pp. 878-884, 1978.

[6] P. F. V. Dessel, L. J. Hornbeck, R. E. Meier, and M. R. Douglass, "A MEMS-based projection display,” Proc. IEEE, vol. 86, no. 8, pp. 16871704, 1988.

[7] T. Chan, E. Myslivets, and J. E. Ford, "2-Dimensional beamsteering using dispersive deflectors and wavelength tuning," Opt. Express, vol. 16, no. 18, pp. 14617-14628, 2008.

[8] J. Kim, M. N. Miskiewicz, S. Serati, and M. J. Escuti, "Nonmechanical laser beam steering based on polymer polarization gratings: design optimization and demonstration," J. Lightwave. Technol., vol.33, no.10, pp. 2068-2077, 2015.

[9] Y. Kurosaka, S. Iwahashi, Y. Liang, K. Sakai, E. Miyai, W. Kunishi, D. Ohnishi, and S. Noda, "On-chip beam-steering photonic-crystal lasers," Nat. Photon., vol. 4, pp. 447-450, 2010.

[10] J. Shi, P. J. Bos, B. Winker, and P. F. McManamon, "Switchable optical phased prism arrays for beam steering," Proc. SPIE, vol. 5553, no. 1, pp. 102-111, 2004.

[11] G. Wang, U. Habib, C. Wang, N. Gomes, Z. Yan, and L. Zhang, "Wavelength-controlled beam steering for optical wireless transmission using an in-fiber diffraction grating," Conference on Lasers and ElectroOptics (Optical Society of America, 2017), paper SF1L.5.

[12] J. Albert, L. Y. Shao, and C. Caucheteur, "Tilted fiber Bragg gratings sensors," Laser Photonics Rev., vol. 7, no. 1, pp. 83-108, 2012.

[13] K. Zhou, G. Simpson, X. Chen, L. Zhang, and I. Bennion, "High extinction ratio in-fiber polarizers based on $45^{\circ}$ tilted fiber Bragg gratings," Opt. Lett., vol. 30, no. 11, pp. 1285-1287, 2005.

[14] Z. Yan, C. Mou, K. Zhou, X. Chen, and L. Zhang, "UV-inscription, polarization-dependent loss characteristics and applications of $45^{\circ}$ tilted fiber gratings," J. Lightwave Technol. vol. 29, no. 18, pp. 2715-2724 2011.

[15] K. Zhou, X. Cheng, Z. Yan, A. Adedotun, and L. Zhang, "Optical spectrum analyzer using a 45-degree tilted fiber grating," in Advanced Photonics Congress, Colorado Springs, Colorado, 2012, p. BW2E.7.

[16] G. Wang, C. Wang, Z. Yan, and L. Zhang, "Highly efficient spectrally encoded imaging using a $45^{\circ}$ tilted fiber grating," Opt. Lett., vol. 41, no. 11, pp. 2398-2401, 2016.

[17] G. Wang, C. Wang. "Diffraction limited optical time-stretch microscopy using an in-fibre diffraction grating," Frontiers in Optics/Laser Science (Optical Society of America, 2017), paper FF2A.5. 